\title{
Researches on the Application of the New improved Analytic Hierarchy Process in the Physical Achievements Inspection System
}

\author{
Shuhe Shao \\ Department of Physical Education, \\ Henan Institute of Science and Technology, \\ Xinxiang, 453003, China P.R \\ qianguoximi@163.com
}

\begin{abstract}
The physical class is always out of the door. This class model is different from the traditional class. As this special characteristic, how to pnsure the weigh of the sports test course achievement evaluation becomes necessary and important the application of AHP makes it possible to carry out the quantity evaluation in the sports research. The study makes a comprehensive evaluation with AHP and puts forward a method for relative analysis and a thereunder for decision-maker.oFom the necessary and sufficient conditions of completely consistent matrix the criterion of consistency has been improved. We create a new improved AHP method, namely (MGAHP (matrix correction analytic hierarchy process). And we apply to to examine the physical class in determining the weights of evaluation indicators bystudents. Examples show that the improved algorithm is better than the original AHP in significantly reduced computation. And this method improves the running speed. The first part is the introduction. The second part is the steps of AHP. The third partis the study on the MCAHP algorithm. The fourth part is the exploration of the MCAHP algorithm. The fifth part is the numerical experiment and the last part is the condusion.
\end{abstract}

Keywords MCAHP, PhysicalAchievements Inspection System, Analysis

\section{Introduction}

Compared with the traditional culture education, the physical education attends the classes outside in most cases. In addition, the number of inspections is less than the traditional culture education. Due to the particularity of the physical education, it becomes a key factor to judge the weights of the student physical test course achievement evaluation indexes.

Yan Linlin, Zhang Wenge and Wu Jingqi analyzed the physical factors of college physical education evaluation system [1]. They used the method of literature and investigation to study the comprehensive evaluation system in colleges and universities at present. They also focused on the analysis of the current situation of the physical indexes which relate to the health status of university students. In addition, they put forward to make full use of the guiding and excitation function to solve the increasingly serious health problems and achieve the comprehensive development for students. Peng Shuolong studied the indicators which affected the sports development factors [2]. He studied and analyzed the most direct and the most significant factors which effected the development of the competitive sports from different levels and different angles. Adopting the method of the stepwise regression to list the optimal equation, he carried on the quantitative analysis of the optimal index which effected the development of competitive sports from the macroscopic, meso and microcosmic levels. Aiming at enriching the sport theory of the qualitative research and providing the scientific basis for formulating policies on 
sports, Liu Houlin and $\mathrm{Bu}$ Deshou explored a new formula of the mean test on the physical statistics [3]. They pointed out that the mean test of the current physical indexes is two different types. And they proposed a new mean test statistic function for the first time according to the type of the probability characteristics.

The investigation of the physical examination course achievement evaluation index is a complex system with multi-factors. However, some problems cannot be established as the mathematical model for the quantitative analysis. Due to the tight time, some problems may not be quantitatively analyzed. We just need to make a preliminary selection and a general assessment. The decision makers face a choice, and it is difficult to make an objective evaluation. This paper attempts to apply the analytic hierarchy process to conduct the comprehensive assessment of the physical examination course achievement evaluation index. Further, this paper seeks a simple and objective evaluation method.

Because of the disadvantages of the traditional AHP method, many scholars put forward an improved AHP method. Some scholars put forward the fuzzy AHP prethod [47]. The method combines the analytic hierarchy process with the fuzzy comprehensive evaluation. This method uses the AHP method to determine the weight of each index in the evaluation system. In addition, it uses the fuzzy comprehensive evaluation method to evaluate the fuzzy indexes. The AHP fuzzy method solves the fuzzy problems perfectly. Later, some scholars proposed an AHP-Entropy method [8-10]. The entropy method is the objective weighting method, and it determines the attribute weights according to the contact degree of each attribute or the size of the information which is provided by each attribute. The AHP-Entropy portfolio analysis method considers the index data and the subjective preference of the index for the decision makers. With the proposed of the grey theory, scholars combine the AHP method with the grey theory and put forward a GreyAHP method [11-13]. This method disposes the decentralized information of the evaluation experts to a weight vector which describes the different grey degrees. On the basis, we make the single value processing. Then, we can get the comprehensive evaluation value of the evaluation system. Because this method combines the grey theory, it can be mainly used to handle the uncertain systems of small sample and poor information.

Constructing the judgment natrix which meets the consistency requirement is one of the key issues of AHP We intend to improve the consistency of the possibility of the judgment matrix and performance of AHP. We revise judgment matrix which does not meet the consistencysto Improve the possibility of the original matrix consistency on the basis of maintenance the raw data. Thereby, we propose MCAHP (matrix correction analytic hierarchy process' method. And we apply this method to the assessment of the physical examination course achievement index. The experimental results show that the MCAHP algorithm car provide a scientific index system and weights for evaluating the examination courses quickly.

\section{The Steps of AHP}

Analytic Hierarchy Process is a method for system analysis. This method is proposed by A.L.Saaty at University of Pittsburgh in 20st century. AHP is a simple, flexible and practical multi criteria decision algorithm. This method carries out quantitative analysis for qualitative problems. Index ranking and the weights determination are accomplished by establishing the hierarchical structure model, constructing judgment matrix for each layer, level sequencing and consistency inspection. This method is suitable for multi object decision. It evaluates the degree of each scheme for multiple impact indexes. When a decision is affected by many factors and these are obvious categories among factors, we can choose AHP. We can also use AHP when each index makes quantitative calculation for the influence on the final evaluation without enough data. The characteristic of this method is that can divide the factors in complex problems into interactional levels and 
make them organized. The method combines judging expert opinion with the objective judgment directly and effectively. Then, this method quantitatively describes the significance of the compared results among factors. At last, we calculate the weights that reflect the orders of importance for factors in each layer.

\subsection{Establishing the Hierarchical Structure Model}

If we make system analysis, we need group all the factors firstly. Each group is a layer. There are three layers. The destination Layer is the purpose of solving the problem. The criterion layer is to achieve the intermediate links that the targets refer. The project layer is the measures or policies to solve the problems. The hierarchy structure model is shown as Figure 1.

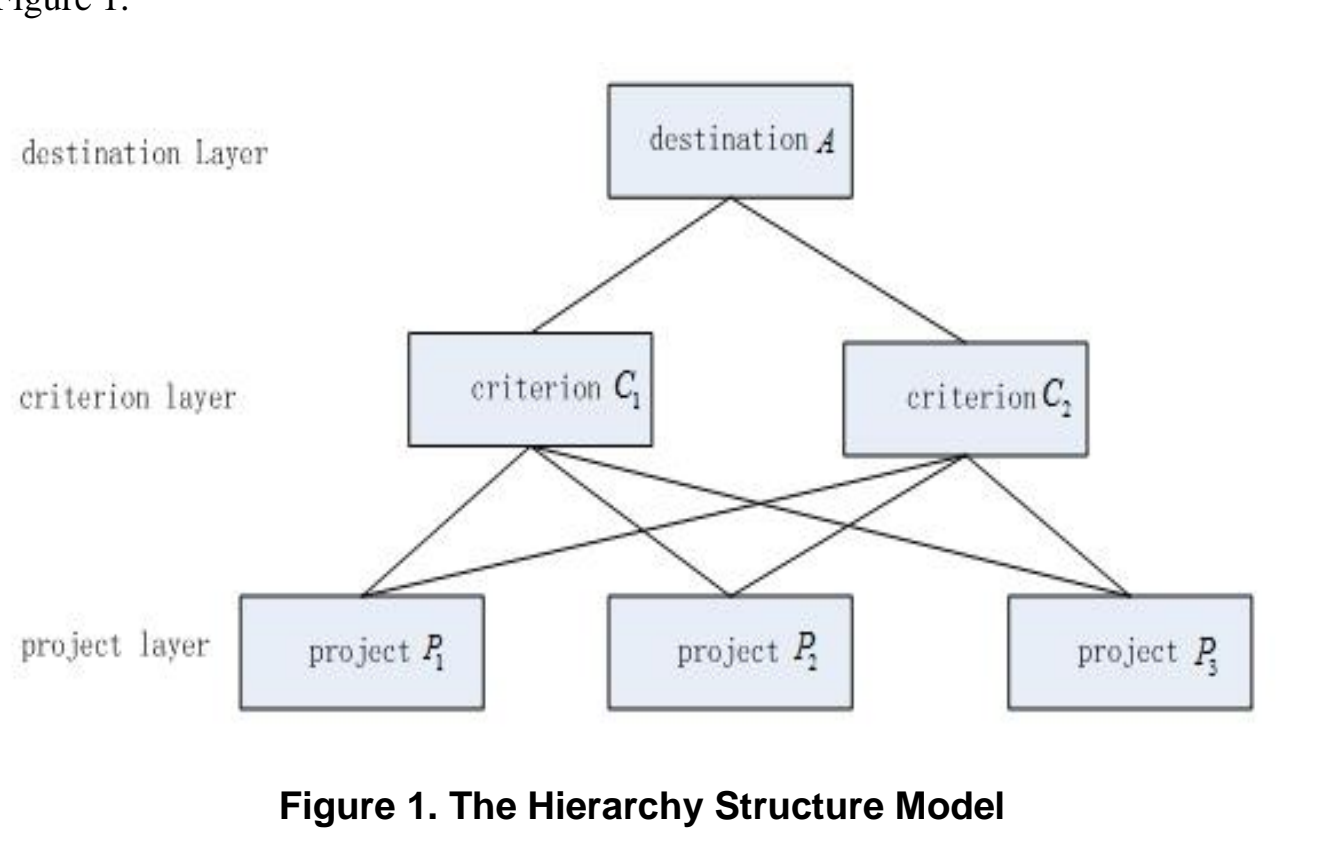

(1) Constructing he hierarehical structure model and establishing the criterion layer and the index layer

(2) Structural comparison matrix

$$
11 A=\left(a_{i j}\right)_{n \times n} \quad(i=1,2, \cdots, n), a_{i j}=1, a_{i j}=1 / a_{j i}
$$

$A$ is the fydgment matrix. We set $a_{i j}$ which shows the relative comparison value of index and $a_{j}$ index.

Table 1. The Judgment Matrix

\begin{tabular}{lllll}
\hline & $a_{1}$ & $a_{2}$ & $\ldots \ldots$ & $a_{i}$ \\
$a_{1}$ & $a_{11}$ & $a_{12}$ & $\ldots \ldots$ & $a_{1 j}$ \\
$a_{2}$ & $a_{21}$ & $a_{22}$ & $\ldots \ldots$ & $a_{2 j}$ \\
$\ldots \ldots$ & $\ldots \ldots$ & $\ldots \ldots$ & $\ldots \ldots$ & $\ldots \ldots$ \\
$a_{j}$ & $a_{j 1}$ & $a_{j 2}$ & $\ldots \ldots$ & $a_{i j}$ \\
\hline
\end{tabular}

Among them, $a_{i j}>0, \frac{1}{a_{i j}}=a_{j i}, a_{i i}=1, a_{i i}=1$

The ratio of Saaty scaling assignment is shown in table 2. 
Table 2. Scale Meaning of Importance Degree

\begin{tabular}{cl}
\hline$a_{i j}$ & Index important degree \\
1 & $a_{i}$ is same important as $a_{j}$ \\
3 & $a_{i}$ is a little more important than $a_{j}$ \\
5 & $a_{i}$ is more important than $a_{j}$ \\
7 & $a_{i}$ is a highly more important than $a_{j}$ \\
8 & $a_{i}$ is a extremely more important than $a_{j}$ \\
$2,4,6,8$ & The importance between $a_{i}$ and $a_{j}$ among the above \\
\hline
\end{tabular}

(3) Judgment matrix $A$ is normalized:

$$
a_{i j}=a_{i j} / \sum_{k=1}^{n} a_{k j} \quad(i=1,2, \cdots, n)
$$

(4) Sum the row of judgment matrix $A$ :

$$
\omega_{i}=\sum_{j=1}^{n} a_{i j}
$$

(5) $\omega_{i}$ is normalized:

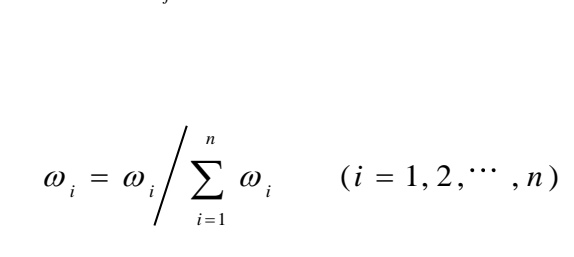

(6) To derive the maximumeigenvalue and its eigenvector according to

(7) Consistency check

We define

$$
C I=\frac{\lambda_{\max }-n}{n-1}
$$

$C I$ is the index of consistency.

When the Judgment matrix has the character of consistency, $C I=0$

If $\lambda_{\max }-7$ is large, $C I$ is large. And the consistency is worse.

For checking whether the judgment matrix has the character of consistency, we compare $C I$ with the index of consistency $R I$ that is shown in table.3.

Table 3. The Index of Consistency from 1-9 Orders

\begin{tabular}{lccccccccc}
\hline order & 1 & 2 & 3 & 4 & 5 & 6 & 7 & 8 & 9 \\
$R I$ & 0.00 & 0.00 & 0.58 & 0.90 & 1.12 & 1.24 & 1.32 & 1.41 & 1.45 \\
\hline
\end{tabular}

\subsection{Calculating the Largest Eigenvalue and Eigenvector}

We apply the approximate method to calculate for simply calculation. There are two methods: One is the sum and product methodand the other one is the root method.

Sum and product method :

(1) Normalizing each line in the judgment matrix. 


$$
a_{i j}=a_{i j} / \sum_{k=1}^{n} a_{k j} \quad(i=1,2, \cdots, n)
$$

(2) Summing each line after normalization

$$
\bar{\omega}_{i}=\sum_{j=1}^{n} a_{i j} \quad(j=1,2, \cdots, n)
$$

(3) Normalizing the vector quantity $\bar{\omega}=\left[\bar{\omega}_{1}, \bar{\omega}_{2}, \cdots, \overline{\omega_{n}}\right]$

$$
\omega=\frac{\bar{\omega}_{i}}{\sum_{j=1}^{n} \bar{\omega}_{i}}, i=1,2, \cdots, n
$$

$\omega$ is eigenvector

Root method:

(1) The factors in the judgment matrix multiply by roy

(2) Turning evolution for products respectively

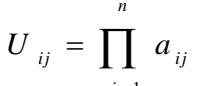

pectively

(3) Normalizing the vectorquantity

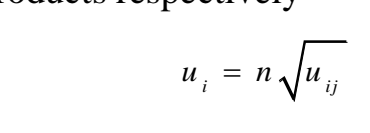

$\omega_{i}$ is eigenvector

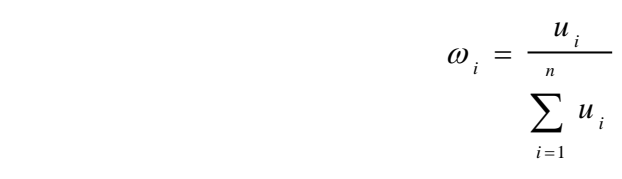

(4) Calculating the largest eigenvalue

$$
\lambda_{\max }=\sum_{i=1}^{n} \frac{\left(A \omega_{i}\right)}{n \omega_{i}}
$$

\section{The Study on the New Modified AHP Algorithm}

\subsection{The Revise of the Judgment Matrix}

If the elements in the judgment matrix meet $a_{i j}>0, a_{i j}=\frac{1}{a_{j i}}, a_{i i}=1(i, j=1,2, \cdots, n)$, the matrix $A$ is called the positive reciprocal matrix.

Definition $1 \mathrm{We}$ assume the judgment matrix is $A=\left(a_{i j}\right)_{n \times n}$. If $\forall i, j, k=1,2, \cdots, n$, there is $a_{i j}=a_{i k} a_{k j}$. We can call the judgment matrix $A$ as the consistent matrix.

The consistent matrix has several properties as follows.

Theorem 1 The sufficient and necessary condition for judging $A=\left(a_{i j}\right)_{n \times n}$ with the characteristic of the consistency is that the maximum eigenvalue of the matrix $A$ is $\lambda_{\max }=n$. The normalized feature vector $\tilde{\omega}=\left(\tilde{\omega}_{1}, \tilde{\omega}_{2}, \cdots \tilde{\omega}_{n}\right)^{T}$ is the weight vector. 
Theorem 2 The sufficient and necessary condition for judging $A=\left(a_{i j}\right)_{n \times n}$ with the characteristic of the consistency is that there has $a_{i j}=a_{i k} a_{k j}$ for $\forall i, j, k=1,2, \cdots, n$.

From the definition 1 and the theorem 2, if the positive reciprocal matrix $A$ is the consistent matrix, there must have

$$
\forall i, j, k=1,2, \cdots, n, a_{i j}=a_{i k} a_{k j} .
$$

We can get the sum for $k$ from formula 1 .

$$
a_{i j}=\frac{1}{n} \sum_{k=1}^{n} a_{i k} a_{k j}
$$

If the positive reciprocal matrix $A$ is the consistent matrix, the sufficient and necessary condition is formula (1). And the formula (2) is the necessary condition. That 15 , if the formula (2) is not infringement, the matrix $A$ may be not the consistent matrix. Therefore, the formula (2) is the precondition for judging the consistent matrix. We construct:

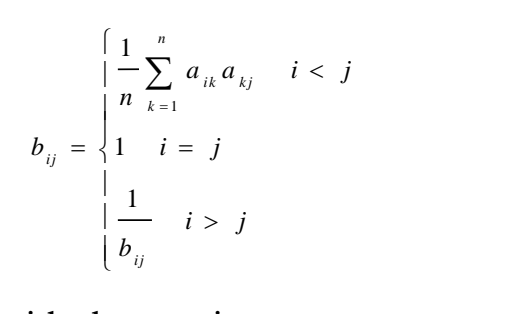

If $A=\left(a_{i j}\right)_{n \times n}$ is not satisfied with the consistency, we use $B=\left(b_{i j}\right)_{n \times n}$ as the modified matrix for the matrix $A$. It improves the possibility to meet the consistency. If $A$ is a consistent matrix, $B=A$.

\subsection{The Improved Consistent Test}

The maximum eigenvalue of the matrix $A$ is $\lambda_{\max }$. We assume that $\tilde{\omega}$ is the corresponding and normalized feature vector of the $\lambda_{\max }$. From the theorem (1), we can know that $\tilde{\omega}\left(\tilde{\omega}, \tilde{\omega}_{2}, \cdots \tilde{\omega}_{n}\right.$, is the hierarchical single-sort weight vector.

$$
\text { 8. } A \tilde{\omega}=\lambda_{\max } \tilde{\omega}
$$

The matrix $A$ is the completely consistent matrix and its maximum eigenvalue is $\lambda_{\max }=n$. If $A$ does not have the complete consistency, $\lambda_{\max }$ is larger than $n$ slightly.

$$
\|A \tilde{\omega}-\lambda \tilde{\omega}\|=\|A \tilde{\omega}-n \tilde{\omega}\|<\varepsilon
$$

We use formula (5) as the test standard to test the consistency for the matrix $A$.

From the consistency ratio $C R=\frac{C I}{R I}=\frac{\lambda_{\max }-n}{(n-1) R I}<0.1$ which put forward by the professor

T.L.Saaty, we can get:

$$
\left|\lambda_{\max }-n\right|<0.1(n-1) R I
$$

Putting the formula (6) into $\|A \tilde{\omega}-n \tilde{\omega}\|=\left|\lambda_{\max }-n\right|^{\circ}\|\tilde{\omega}\| \leq\left|\lambda_{\max }-n\right|$ we get the formula as follows.

$$
\begin{gathered}
\varepsilon=0.1(n-1) R I \\
|A \tilde{\omega}-n \tilde{\omega}|<0.1(n-1) R I
\end{gathered}
$$


We use formula (8) to test the satisfactory consistency of the matrix. It can omit to solve the eigenvalue of the matrix $A$. We only need to calculate the ranking vector. It simplifies the operation and enhances the running speed.

\subsubsection{The Consistent Test Sample of the Matrix: We know that}

$$
A=\left\{\begin{array}{llll}
1 & 8 & 9 & \\
1 / 8 & 1 & 9
\end{array} \mid\right.
$$

We get the normalized feature vector by using the square method.

and

$$
\tilde{\omega}=\left[\begin{array}{l}
0.715 \\
0.245 \\
0.049
\end{array}\right]
$$

$$
\varepsilon=0.1(n-1) R I=0.116^{\circ} \text {. }
$$

However, $\|A \tilde{\omega}-n \tilde{\omega}\|=\|A \tilde{\omega}-3 \tilde{\omega}\|=1,16+>0.116$ is (not satisfied with the formula (8). Therefore, the matrix does not have the satisfactory consistency. According to the formula (3) we revise the matrix $A$ and get the following matrix.

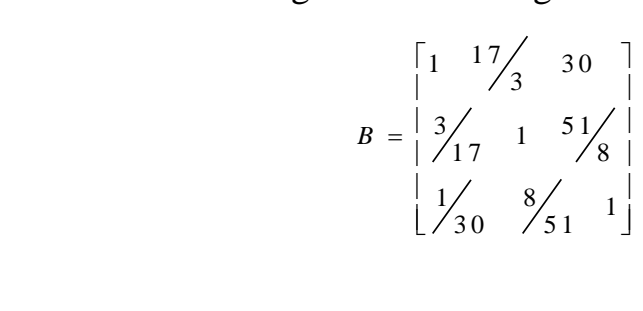

The normalized eigenvector is

$$
\tilde{\omega}^{*}=\left[\begin{array}{l}
0.820 \\
0.154 \\
0.026
\end{array}\right]
$$

The tested formula is $\left\|B \tilde{\omega}^{*}-n \tilde{\omega}^{*}\right\|=\left\|B \tilde{\omega}^{*}-3 \tilde{\omega}^{*}\right\|=0.013<0.116$. Therefore, the revised matrix has the characteristic of the satisfactory consistency.

3.2.2. The Calculated Steps of the MCAHP Algorithm: The first step is analyzing problems and establishing a hierarchical analysis model. The model contains the target layer, the attribute layer and the scheme layer.

The second step is adopting the 1 9 scaling method that is proposed by professor T.L.Saaty to construct the judgment matrix at each layer.

The third step is using the square root method to calculate the priority weights of each layer elements.

The fourth step is using the formula (8) to test the consistency of the judgment matrix. If the matrix meets the consistency, we execute the fifth step. If the matrix does not meet the consistency, we revise the judgment matrix by using the formula (3) and execute the third step.

The fifth step is calculating the total order weighs of each scheme. 


$$
\tilde{\omega}_{i}=\sum_{j=1}^{m} \tilde{\omega}_{j} \omega_{i}^{j}
$$

Among them, $\tilde{\omega}_{i}^{j}$ is the ranking weight of the scheme $i$ in attribute $j \cdot \tilde{\omega}_{j}^{c}$ is the weight of the attribute $j . m$ is the number of the attributes.

\section{The Exploration of the MCAHP Algorithm}

Compared with the original AHP algorithm, the MCAHP algorithm in this paper is simpler. The MCAHP algorithm revises the judgment matrix which cannot satisfy the consistency. Therefore, the new algorithm avoids the progress of reinvestigation. And it also collects data and constructs the judgment matrix while it shortens the needed time to resolve the problems. In addition, it also saves the manpower and the financial resources. Because of the improvement of the consistency criterion, we can use the solved normalized feature vector and the matrix directly to judge the consistency. The MCAHP algorithm avoids the calculation of the maximum eigenyalure.

After a lot of practices prove, the MCAHP algorithm only heeds one time revise. So it satisfies the consistent condition for the judgment matrix which cannot meet the consistency. After one-time revises, we can revise the matric again according to the improved AHP algorithm if the matrix still cannot satisfy with the consistency. In general, an algorithm can terminate running and get an effective result after the finite times(less 20 times).

In this paper, the improved algoritam, ean also apply the exponential scale method, three standard method $([0,1,2])$ and other methods. It is also valid for revising the judgment matrix and tests the consistency. The operation results of the MCAHP algorithm are the same with the original AHP algorithmiresults.

\section{Using the MCAHP Algorithm to Ensure the Weight of the Sports Test Course Achievement Ev́luation}

We establish the index system for ensuring the physical examination course (figure 2) through extensively collecting the opinions of the experts, teachers and students. We select the pairwise comparison method to construct the judgment matrix at each level by providing a large number of questionnaires. For different judgment matrixes that are obtained from the same index, we use the geometric mean method to get the comprehensive judgment matrix of the index. At last, we use the MCAHP algorithm to solve the weights of each index in this paper. The total order weight is shown in Figure 2.

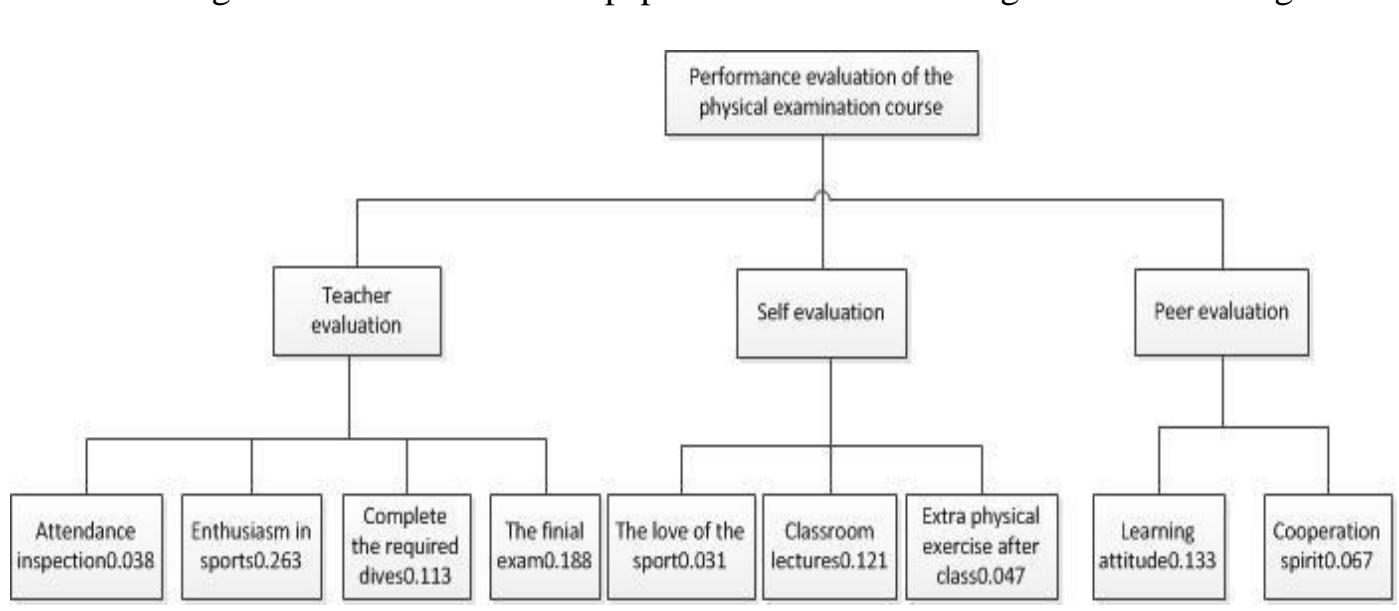

Figure 2. The Physical Examination Course Achievement Evaluation Index System and the Total Order Weights 
Table 4. The Teachers' Evaluation Matrix at Scheme Layer

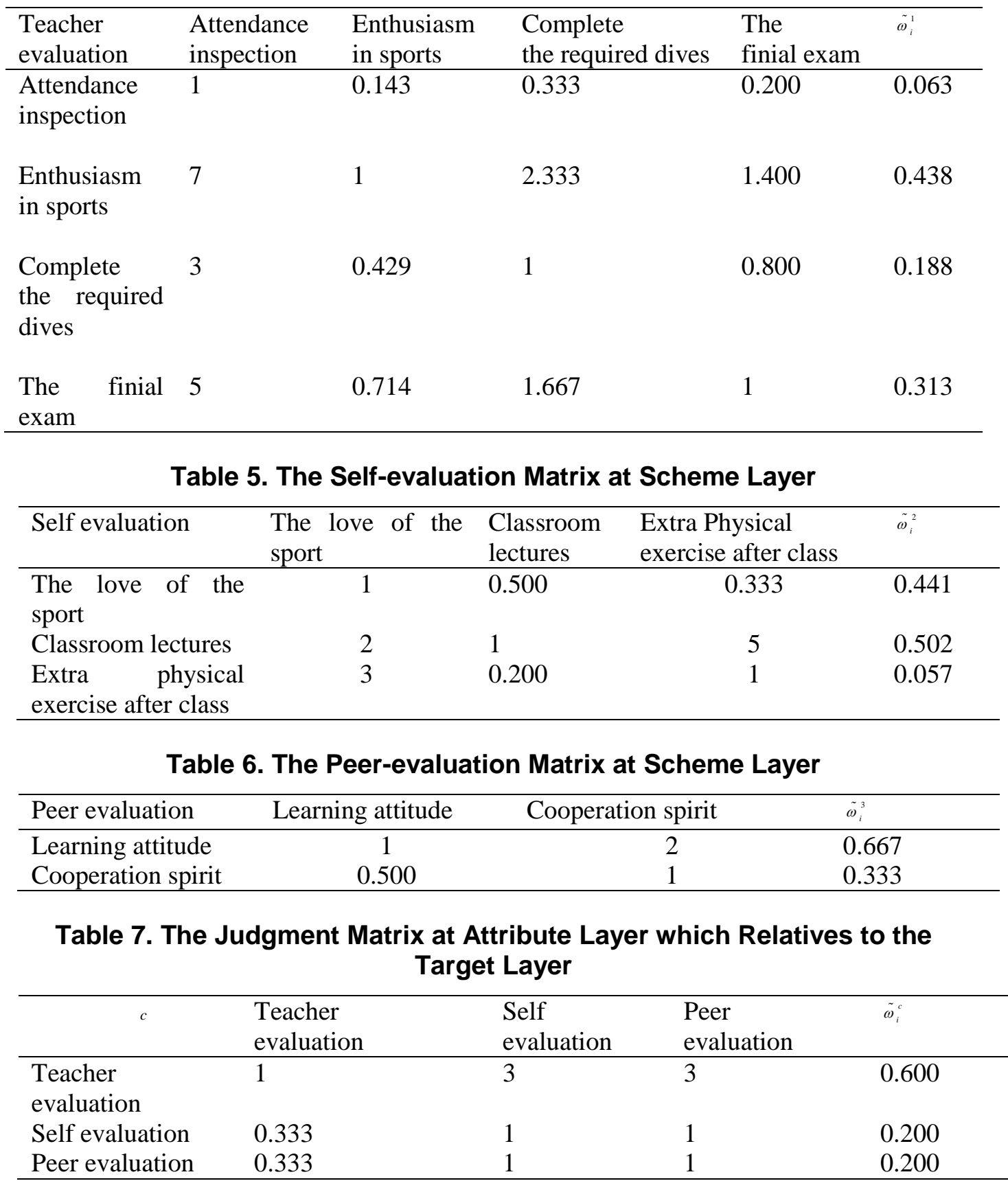

Among them, the relative attributive judgment matrix of the student self-assessment is $A_{2}$. The hierarchical single sequencing vector $\tilde{\omega}_{2}=(0.156,0.607,0.237)^{T}$, $\left\|A_{2} \tilde{\omega}_{2}-n \tilde{\omega}_{2}\right\|=0.382>0.1(n-1) R I=0.116$ is not satisfied with the consistency. The hierarchical single sequencing weight $\tilde{\omega}_{i}^{2}=(0.441,0.502,0.057)^{T},\left\|B_{2} \tilde{\omega}_{i}^{2}-n \tilde{\omega}_{i}^{2}\right\|=0.022<0.116$ which corresponds to the modified matrix $B_{2}$ is satisfied with the consistency.

From the sequencing results, we can see that the main factors which affect the performances are the enthusiasm in sports and the final exam. The influences of the attendance inspection and completing the required dives are weaker. This result is same to the original AHP algorithm basically. However, the running speed of the MCAHP 
algorithm is faster while it keeps the original data which are provides by the experts at the maximum.

\section{Conclusion}

Compared with the traditional culture education, the physical education attends classes outside in most cases. In addition, the number of inspection is less than the traditional culture education. Due to the particularity of the physical education, it becomes a key factor to judge the weights of the student physical test course achievement evaluation indexes.

Because the subsystem of the sports management system is affected by many factors, using the analytic hierarchy process has obvious advantages. The analytic hierarchy process requirements the data little accurate and its calculation is simple. It is suitable) for solving the decision-making problems which are difficult to analyze by using the quantitative method. AHP is an effective tool to achieve the scientific decision for the complex society and the economic system.

In this paper, we apply the MCAHP proposes to determine the index weights of student examination course evaluation. This method enhancer the possibility of the consistency for the judgment matrix and the universality of the sequencing results application. The use of the new improved AHP algorithm provides the scientific index system and the weights for the evaluation of the test course achievement conveniently. The improved algorithm can also be applied to other weight decision problems this new method has a broad development space and an applicable prospect.

\section{References}

[1] L.-1. Yan, W.-g. Zhang and J.-y.Wu, "Analysis of Physical Education as the Influence Factor in Synthetic Test System of the Colleges and Universities", Journal of Harbin Institute of Physical Education, vol. 27, no. 2, (2009), pp. 58-61

[2] Y. L. Peng, "Research on the indexes that affected the competitive sports development", Journal of Xi'an Institute of Physical Education, vol. 1, no. 8, (1991), pp. 47-58.

[3] L. Housheng and B Deshou, "On a new formular of testing the sample mean in statistics of physical culture", Journal of Central China Nornal University, vol. 23, no. 3, (1989), pp. 427-432.

[4] K. Sachin, R. K. Patil, "A fuzzy AHP-TOPSIS framework for ranking the solutions of Knowledge Management adoption in Supply Chain to overcome its barriers", Expert Systems with Applications, vol. 41, no. 2, (2014), pp. 679-693.

[5] A. Calabrese, R. Costa and T. Menichini, "Using Fuzzy AHP to manage Intellectual Capital assets: An application to the ICT service industry", Expert Systems with Applications, vol. 40, no. 9, (2013), pp. 3747-3755.

[6] G. in Bâuük zkan, Gizem if i. A combined fuzzy AHP and fuzzy TOPSIS based strategic analysis of electronic service quality in healthcare industry, Expert Systems with Applications, vol. 39, (2012), pp. 2341-2354.

[7] L. Gaoa and A. Hailu, "Identifying preferred management options: An integrated agent-based pecreational fishing simulation model with an AHP-TOPSIS evaluation method", Ecological Modelling, vol. 249, (2013), pp. 75-83.

[8] S.-W. Hsiao and J.-R. Chou, "A Gestalt-like perceptual measure for home page design using a fuzzy entropy approach”, Int. J. Human-Computer Studies, vol. 64, (2006), pp. 137-156.

[9] M. Xia and Z. Xu, "Entropy/cross entropy-based group decision making under intuitionistic fuzzy environment", Information Fusion, vol. 13, (2012), pp. 31-47.

[10] T.-Y. Chena and C.-H. Li, "Objective weights with intuitionistic fuzzy entropy measures and computational experiment analysis", Applied Soft Computing, vol. 11, (2011), pp. 5411-5423.

[11] H. Yin and B.-z. Li, "Evaluation of High New-Tech Outcome's Transformative Comprehensive Value Based on AHP-GCA method", Operations Research and Management Science, vol. 18, no. 5, (2009), pp. 107-115.

[12] Q. Zhang, P. Hua and J. Zhang, "Improved AHP Assessment and Grey Relational Analysis Model for Optimizing Waterworks Sludge Treatment Process", Journal of Civil, Architectural and Environmental Engineering, vol. 32, no. 3, (2012), pp. 133-144.

[13] F. Shao, W. Deng, F.-j. Yi and X.-b. Liu, "Comprehensive evaluation method of metropolitan public transportation system based on analysis hierarchical process and grey theory", Journal of PLA University of Science and Technology, vol. 10, no. 6, (2009), pp. 536-541. 


\section{Author}

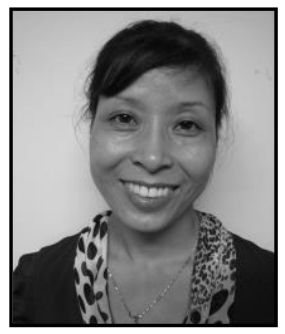

Shuhe Shao, she received her M.Ed. in sports science (2005) from University. Now he is a lecturer of sports science at Department of Physical Education, University. Since 2009 she is Senior Member of IEEE. His current research interests include different aspects of the application of computer model in the sports.

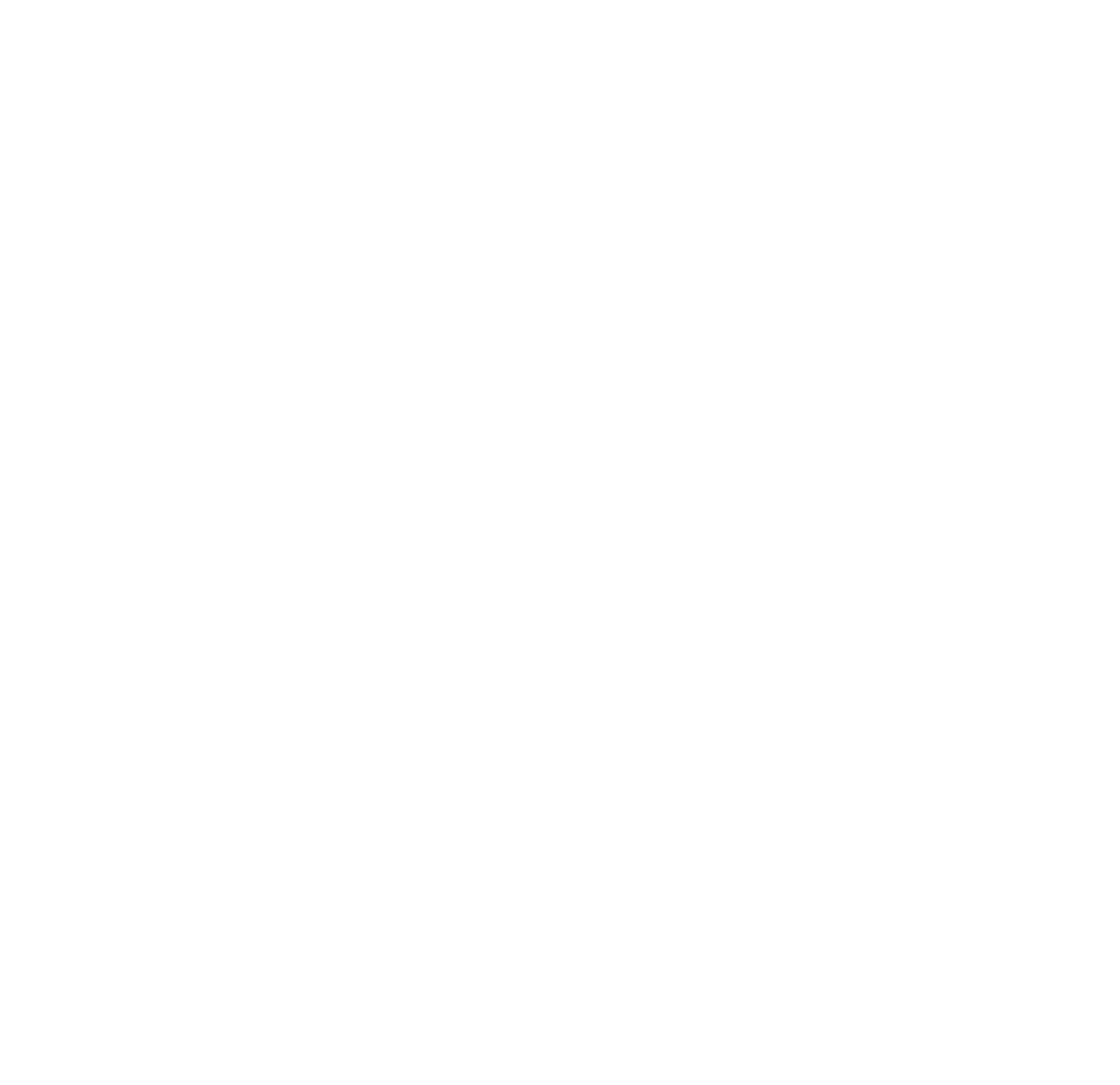


International Journal of Multimedia and Ubiquitous Engineering

Vol.9, No.8 (2014)

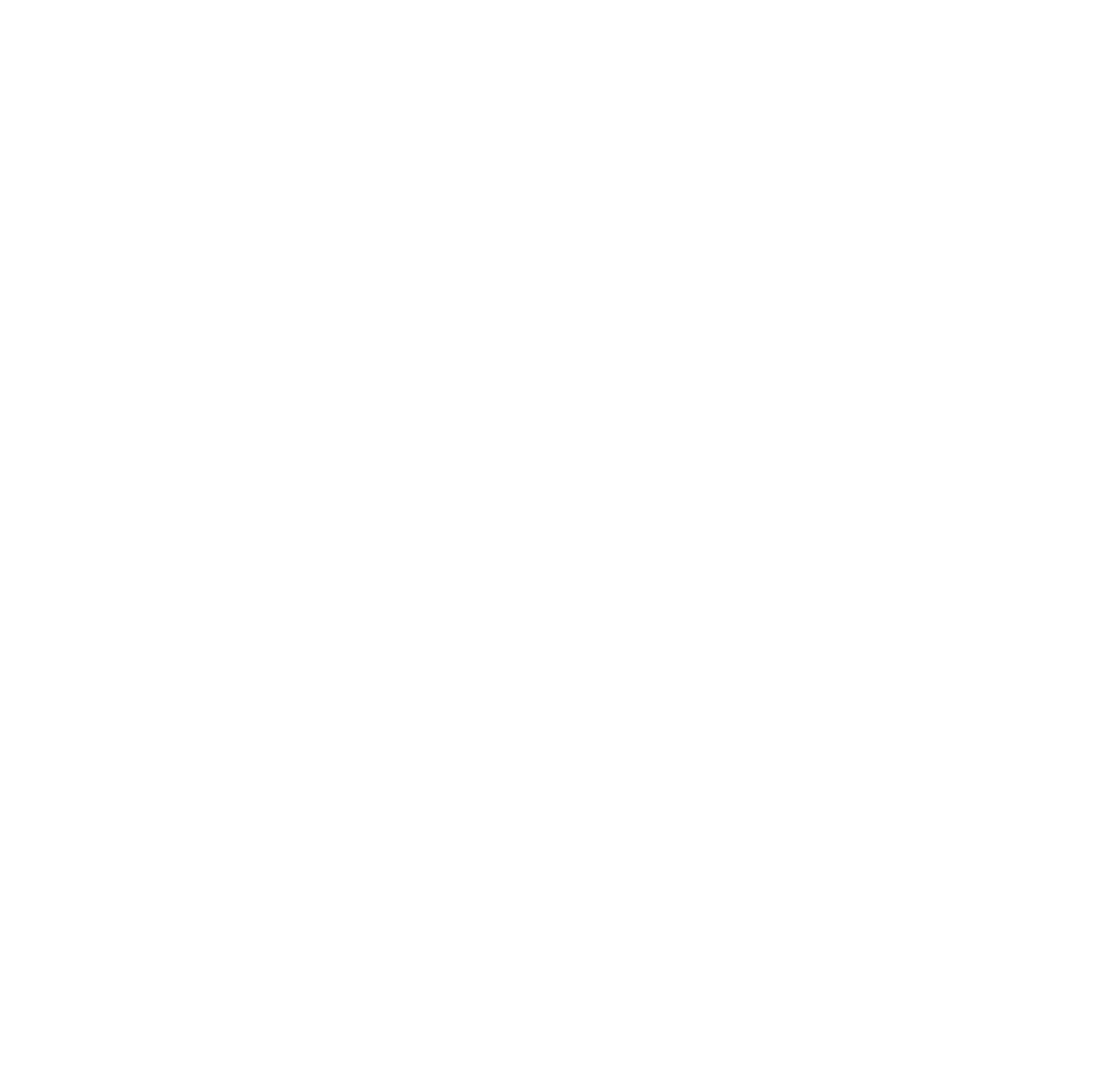

